\title{
Analytical model of strange star in the low-mass X-ray binary 4U 1820-30
}

\author{
Mehedi Kalam ${ }^{1, a}$, Farook Rahaman ${ }^{2, b}$, Sajahan Molla ${ }^{1, c}$, Md. Abdul Kayum Jafry ${ }^{3, d}$, Sk. Monowar Hossein ${ }^{4, e}$ \\ ${ }^{1}$ Department of Physics, Aliah University, Sector-V, Salt Lake, Kolkata 700091, India \\ ${ }^{2}$ Department of Mathematics, Jadavpur University, Kolkata 700 032, West Bengal, India \\ ${ }^{3}$ Department of Physics, Shibpur Dinobundhoo Institution (College), Howrah 711102, West Bengal, India \\ ${ }^{4}$ Department of Mathematics, Aliah University, Sector-V, Salt Lake, Kolkata 700091, India
}

Received: 13 February 2014 / Accepted: 6 July 2014 / Published online: 29 July 2014

(C) The Author(s) 2014. This article is published with open access at Springerlink.com

\begin{abstract}
In this article, we propose a model for a realistic strange star under Tolman VII metric (Tolman, Phys Rev 55:364, 1939). Here the field equations are reduced to a system of three algebraic equations for anisotropic pressure. Mass, central density and surface density of strange star in the low-mass X-ray binary $4 \mathrm{U} 1820-30$ are matched with the observational data according to our model. Strange materials clearly satisfy the stability condition (i.e. sound velocities $<1)$ and TOV equation. Here also the surface redshift of the star is found to be within a reasonable limit.
\end{abstract}

\section{Introduction}

Compact objects have attracted great attention for a long time. Several researchers [1-10] investigated compact stars analytically or numerically. Stars, in general, evolve by burning lighter elements into heavier nuclei from the time of birth. In the end of nuclear burning white dwarfs, neutron stars, quark stars, dark stars and eventually black holes may form due to strong gravity. To include the effects of local anisotropy, Bowers and Liang [11] stressed the importance of local anisotropic equations of state for a relativistic fluid sphere. They showed that anisotropy may have effects on such parameters as the maximum equilibrium mass and the surface redshift. In a stellar system, Ruderman [12] argued that, in the very high density range $\left(\sim 10^{15} \mathrm{gm} \mathrm{cm}^{-3}\right)$, nuclear matter may have anisotropic features and the nuclear interaction should be treated relativistically. The anisotropy in

\footnotetext{
a e-mail: kalam@iucaa.ernet.in

be-mail: rahaman@iucaa.ernet.in

c e-mail: sajahan.phy@gmail.com

de-mail: akjafry@yahoo.com

e e-mail: hossein@iucaa.ernet.in
}

matter indicates that the radial pressure $\left(p_{r}\right)$ is not the same as the tangential pressure $\left(p_{t}\right)$. A star becomes anisotropic if its matter density exceeds the nuclear density $[11,13,14]$. This phenomenon may occur in the presence of a solid core, a phase transition, a electromagnetic field etc. 4U 1820-30 resides in the globular cluster NGC 6624. It is an ultracompact binary and has an orbital period of 11.4 min [15]. During Rossi X-ray Timing Explorer (RXTE) observations, it has been observed that $4 \mathrm{U} 1820-30$ exhibits a superburst. Possibly this is due to burning of a large mass of carbon [16]. The 4U 1820-30 exhibits a super-burst, however, these strange stars may be made of chemically equilibrated strange matter. Scientists are searching for a matter distribution which should be incorporated in the energy momentum tensor to describe the strange stars. This paper depicts how this is accomplished mathematically and discusses the consequences of the properties of the strange stars. There are many high mass stars, found in different types of pulsar binaries. In these cases the masses rely on the observation of the periastron advance, which is believed to be due to general relativistic effects only rather than other effects such as rotationally and tidally induced quadrupoles. One of the useful tools for determining the masses of the compact stars is $\mathrm{X}$-ray eclipses. The binary eclipses are approximated analytically by assuming that the companion star is spherical with effectively a Roche lobe radius.

In 1939, Tolman [17] proposed static solutions for a sphere of fluid. In that article, he pointed out that due to the complexity of the VIIth solution (among the eight different solutions), it is not a feasible one for physical consideration [there was a misprint in the Tolman solution VII (4.7) but that does not affect the original solution]. It seems to be due to the complicated nature of the solution that he was not able to provide more physical properties of the solution. Rather we say that he did not try to explore physics of his solution VII due to the 
complexity of the solution. We thought this solution may illuminate some physics. In this work, we have shown that this solution would be interesting in the sense that this solution corresponds to the interior of strange stars. We, here, want to check the feasibility of our model by taking the Tolman solution VII.

Motivated by the above fact, we are specifically interested in modelling the strange star in the low-mass X-ray binary 4U 1820-30. We compare our measurements of mass, radius, central density, surface density and surface redshift with the strange star in the low-mass X-ray binary $4 \mathrm{U} 1820-30$ and it is found to be consistent with the standard data [18].

The density within the strange stars is normally beyond the nuclear matter density. The theoretical advance in the last few decades indicates that pressures within the stars are anisotropic. Thus one would expect that anisotropy plays a major role in modelling these stars.

We consider that the interior spacetime geometry of the strange star is Tolman VII type and try to investigate the matter distributions which produce this spacetime. Our calculations show that the matter distribution that produces a Tolman VII type spacetime geometry should be anisotropic. This helps us in modelling the strange star which is anisotropic in nature as the density within the strange stars is normally beyond the nuclear matter density.

In this work, we choose the interior spacetime geometry of the strange star to be Tolman VII type and try to investigate the matter distributions which produce this spacetime. We assume only a Tolman VII spacetime for modelling the strange stars. The other solutions of Tolman are not interesting for us as far as we have learned in our studies [17]. In the Tolman I solution, $e^{v}=$ constant, i.e. the redshift function is constant and therefore not interesting. Tolman II corresponds to the Schwarzschild-de Sitter solution. For the Tolman III solution, the energy density is constant and therefore not interesting. In Tolman IV and V, the redshift functions are very specific and therefore not interesting. In Tolman VI, the coefficient of $\mathrm{d} r^{2}$ has been taken as constant. Therefore we do not consider it. The Tolman VIII solution,

$\mathrm{d} s^{2}=e^{-\lambda}\left[B^{2} r^{2 b} \mathrm{~d} t^{2}-\mathrm{d} r^{2}-r^{2} e^{\lambda} \mathrm{d} \theta^{2}+\sin ^{2} \theta \mathrm{d} \phi^{2}\right]$,

is conformally related to the metric whose redshift function is very specific (polynomial function of $r$ ). So, we discard it.

We organize our paper as follows.

In Sect. 2, we provide the basic equations in connection to the Tolman VII metric. In Sect. 3, we study the physical behaviours of the star, namely, anisotropic behaviour, matching conditions, TOV equations, energy conditions, stability and mass-radius relation and surface redshift in different sub-sections. The article concludes with a short discussion.

\section{Interior solution}

We assume that the interior spacetime of a star is described by the metric

$$
\begin{aligned}
\mathrm{d} s^{2}= & -B^{2} \sin ^{2} \ln \sqrt{\frac{\sqrt{1-\frac{r^{2}}{R^{2}}+4 \frac{r^{4}}{A^{4}}}+2 \frac{r^{2}}{A^{2}}-\frac{1}{4} \frac{A^{2}}{R^{2}}}{C}} \mathrm{~d} t^{2} \\
& +\left(1-\frac{r^{2}}{R^{2}}+4 \frac{r^{4}}{A^{4}}\right)^{-1} \mathrm{~d} r^{2}+r^{2} \mathrm{~d} \Omega^{2}
\end{aligned}
$$

where $R, C, A, B$ are constants. Such a type of metric as (1) was proposed by Tolman [17] (known as the Tolman VII metric) to develop a viable model for a star. We assume that the energy-momentum tensor for the interior of the star has the standard form,

$T_{v}^{\mu}=\left(-\rho, p_{r}, p_{t}, p_{t}\right)$,

where $\rho$ is the energy density, $p_{r}$ and $p_{t}$ are the radial and transverse pressure, respectively. Einstein's field equations accordingly are obtained as $(c=1, G=1)$

$$
\begin{aligned}
& 8 \pi \rho=\left(1-\frac{r^{2}}{R^{2}}+4 \frac{r^{4}}{A^{4}}\right)\left[\frac{\lambda^{\prime}}{r}-\frac{1}{r^{2}}\right]+\frac{1}{r^{2}}, \\
& 8 \pi p_{r}=\left(1-\frac{r^{2}}{R^{2}}+4 \frac{r^{4}}{A^{4}}\right)\left[\frac{v^{\prime}}{r}+\frac{1}{r^{2}}\right]-\frac{1}{r^{2}}, \\
& \text { and } 8 \pi p_{t}=\frac{1}{2}\left(1-\frac{r^{2}}{R^{2}}+4 \frac{r^{4}}{A^{4}}\right) \\
& \times\left[v^{\prime \prime}+\frac{v^{\prime}-\lambda^{\prime}}{r}+\frac{\nu^{\prime 2}-\lambda^{\prime} v^{\prime}}{2}\right],
\end{aligned}
$$

where

$$
\begin{aligned}
e^{\lambda}= & \left(1-\frac{r^{2}}{R^{2}}+4 \frac{r^{4}}{A^{4}}\right), \\
e^{\nu}= & B^{2} \sin ^{2} \ln \sqrt{\frac{\sqrt{1-\frac{r^{2}}{R^{2}}+4 \frac{r^{4}}{A^{4}}}+2 \frac{r^{2}}{A^{2}}-\frac{1}{4} \frac{A^{2}}{R^{2}}}{C},} \\
\lambda^{\prime}= & \frac{\left(2 \frac{r}{R^{2}}-16 \frac{r^{3}}{A^{4}}\right)}{\left(1-\frac{r^{2}}{R^{2}}+4 \frac{r^{4}}{A^{4}}\right)}, \\
v^{\prime}= & \frac{\left(1-\frac{r^{2}}{R^{2}}+4 \frac{r^{4}}{A^{4}}\right){ }^{-1 / 2}\left(-\frac{r}{2 R^{2}}+4 \frac{r^{3}}{A^{4}}\right)+2 \frac{r}{A^{2}}}{\left(\sqrt{\left.1-\frac{r^{2}}{R^{2}}+4 \frac{r^{4}}{A^{4}}+2 \frac{r^{2}}{A^{2}}-\frac{1}{4} \frac{A^{2}}{R^{2}}\right)}\right.} \\
& \times 2 \cot \ln \sqrt{\frac{\sqrt{1-\frac{r^{2}}{R^{2}}+4 \frac{r^{4}}{A^{4}}}+2 \frac{r^{2}}{A^{2}}-\frac{1}{4} \frac{A^{2}}{R^{2}}}{C}}
\end{aligned}
$$


and

$$
\begin{aligned}
& v^{\prime \prime}=\left[\left(\sqrt{1-\frac{r^{2}}{R^{2}}+4 \frac{r^{4}}{A^{4}}}+2 \frac{r^{2}}{A^{2}}-\frac{1}{4} \frac{A^{2}}{R^{2}}\right)^{-1}\right. \\
& \times\left\{-2\left(1-\frac{r^{2}}{R^{2}}+4 \frac{r^{4}}{A^{4}}\right)^{-3 / 2}\left(-\frac{r}{2 R^{2}}+4 \frac{r^{3}}{A^{4}}\right)^{2}\right. \\
& \left.+\left(1-\frac{r^{2}}{R^{2}}+4 \frac{r^{4}}{A^{4}}\right)^{-1 / 2}\left(-\frac{1}{2 R^{2}}+\frac{12 r^{2}}{A^{4}}\right)+\frac{2}{A^{2}}\right\} \\
& -2\left(\sqrt{1-\frac{r^{2}}{R^{2}}+4 \frac{r^{4}}{A^{4}}}+2 \frac{r^{2}}{A^{2}}-\frac{1}{4} \frac{A^{2}}{R^{2}}\right)^{-2} \\
& \left.\times\left\{\left(1-\frac{r^{2}}{R^{2}}+4 \frac{r^{4}}{A^{4}}\right)^{-1 / 2}\left(-\frac{r}{2 R^{2}}+4 \frac{r^{3}}{A^{4}}\right)+\frac{2 r}{A^{2}}\right\}^{2}\right] \\
& \times 2 \cot \ln \sqrt{\frac{\sqrt{1-\frac{r^{2}}{R^{2}}+4 \frac{r^{4}}{A^{4}}}+2 \frac{r^{2}}{A^{2}}-\frac{1}{4} \frac{A^{2}}{R^{2}}}{C}} \\
& -2\left[\frac{\left(1-\frac{r^{2}}{R^{2}}+4 \frac{r^{4}}{A^{4}}\right)^{-1 / 2}\left(-\frac{r}{2 R^{2}}+4 \frac{r^{3}}{A^{4}}\right)+\frac{2 r}{A^{2}}}{\sqrt{1-\frac{r^{2}}{R^{2}}+4 \frac{r^{4}}{A^{4}}}+2 \frac{r^{2}}{A^{2}}-\frac{1}{4} \frac{A^{2}}{R^{2}}}\right. \\
& \left.\times \operatorname{cosec} \ln \sqrt{\frac{\sqrt{1-\frac{r^{2}}{R^{2}}+4 \frac{r^{4}}{A^{4}}}+2 \frac{r^{2}}{A^{2}}-\frac{1}{4} \frac{A^{2}}{R^{2}}}{C}}\right]^{2} \text {. }
\end{aligned}
$$

\section{Analysis of physical behaviour}

In this section we will discuss the following features of the anisotropic strange star.

\subsection{Density and pressure behaviour of the star}

Now from Eqs. (3) and (6) we get

$$
\rho=\frac{1}{8 \pi}\left(\frac{3}{R^{2}}-20 \frac{r^{2}}{A^{4}}\right) .
$$

Therefore, $\quad \rho_{0}=\frac{3}{8 \pi R^{2}}$,

$\rho_{b}=\frac{1}{8 \pi}\left(\frac{3}{R^{2}}-20 \frac{b^{2}}{A^{4}}\right)$,

where we have assumed that $b$ is the radius of the star and $\rho_{0}$ and $\rho_{b}$ is the matter density at the centre and surface of the star.

Now we will check whether at the centre of the star matter density dominates or not. We see that

$$
\begin{aligned}
& \frac{\mathrm{d} \rho}{\mathrm{d} r}=-\frac{5 r}{\pi A^{4}}<0, \\
& \frac{\mathrm{d} \rho}{\mathrm{d} r}(r=0)=0, \\
& \frac{\mathrm{d}^{2} \rho}{\mathrm{d} r^{2}}(r=0)=-\frac{5}{\pi A^{4}}<0 .
\end{aligned}
$$

Clearly, at the centre of the star, density is maximum and it decreases radially outward.

Similarly, from Eq. (4), we get

$$
\frac{\mathrm{d} p_{r}}{\mathrm{~d} r}=\frac{\frac{8 r}{A^{4}}+v^{\prime}\left(12 \frac{r^{2}}{A^{4}}-\frac{1}{r^{2}}-\frac{1}{R^{2}}\right)+v^{\prime \prime}\left(4 \frac{r^{3}}{A^{4}}+\frac{1}{r}-\frac{r}{R^{2}}\right)}{8 \pi}<0 .
$$

Now, at the centre $(r=0)$,

$\frac{\mathrm{d} p_{r}}{\mathrm{~d} r}(r=0)=0$

and $\frac{\mathrm{d}^{2} p_{r}}{\mathrm{~d} r^{2}}(r=0)=<0$.

Therefore, at the centre, we also see that the radial pressure is maximum and it decreases from the centre towards the boundary. Thus, the energy density and the radial pressure are well behaved in the interior of the stellar structure. Variations of the energy density and two pressures have been shown in Figs. 1 and 2, respectively.

The anisotropic parameter $\Delta(r)=\left(p_{t}-p_{r}\right)$, representing the anisotropic stress, is given by Fig. 3. The 'anisotropy' will be directed outward when $p_{t}>p_{r}$, i.e. $\Delta>0$, and inward when $p_{t}<p_{r}$ i.e. $\Delta<0$. It is apparent from Fig. 3 for our model that a repulsive 'anisotropic' force $(\Delta>0)$ allows for the construction of more massive distributions.

The dimensionless quantity $\omega(r)=\frac{p_{r}+2 p_{t}}{3 \rho}$ determines a measure of the equation of state. The plot (Fig. 4) for $\omega(r)$

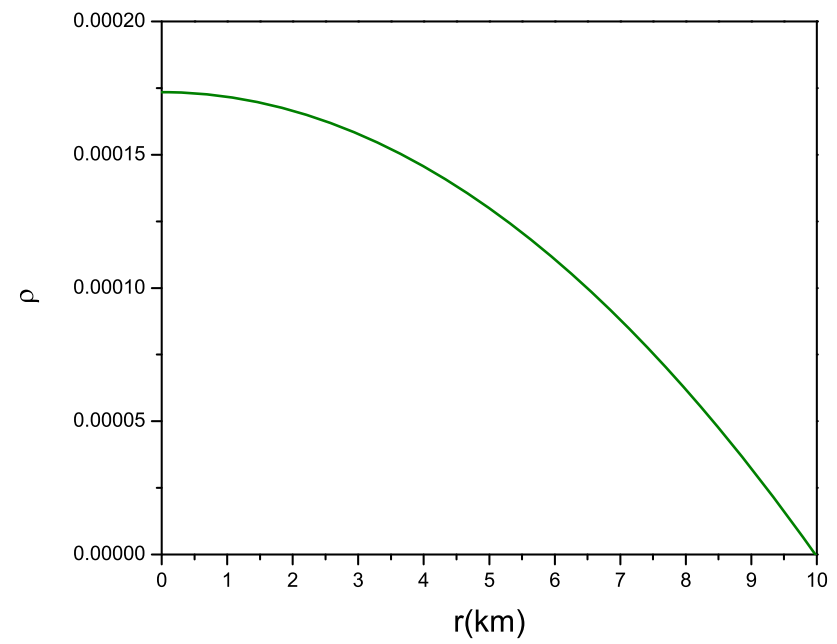

Fig. 1 Variation of the energy density $(\rho)$ at the stellar interior of the strange star. We have taken the numerical values of the parameters as $b=10, R=26.25, A=25.999, C=0.05391$ 


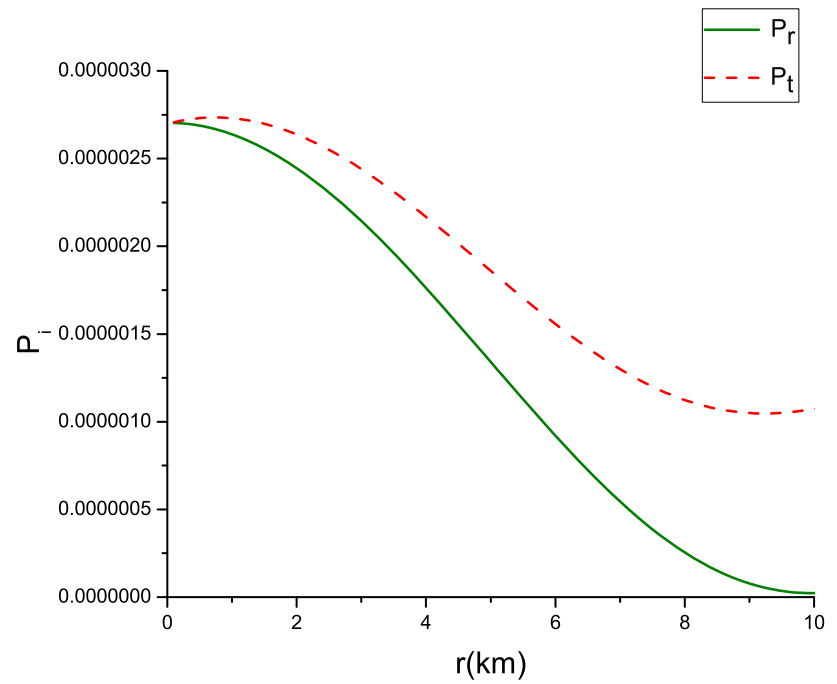

Fig. 2 Variation of the radial and transverse pressure at the stellar interior of the strange star. We have taken the numerical values of the parameters as $b=10, R=26.25, A=25.999, C=0.05391$

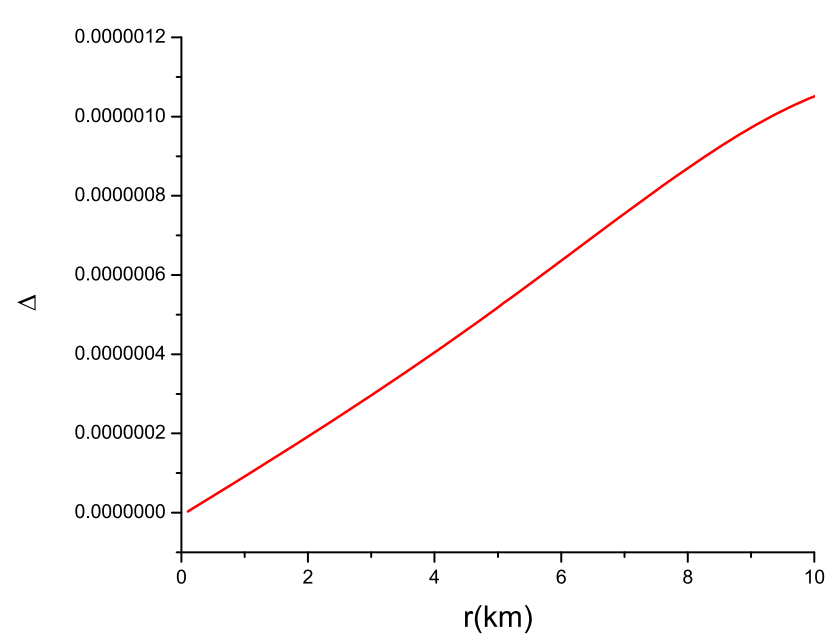

Fig. 3 Effective anisotropic behaviour $\Delta(r)$ at the stellar interior of the strange star. We have taken the numerical values of the parameters as $b=10, R=26.25, A=25.999, C=0.05391$

shows that the equation of state parameter is less than unity within the interior of the strange star.

\subsection{Matching conditions}

The interior metric of the star should be matched to the Schwarzschild exterior metric at the boundary $(r=b)$,

$\mathrm{d} s^{2}=-\left(1-\frac{2 M}{r}\right) \mathrm{d} t^{2}+\left(1-\frac{2 M}{r}\right)^{-1} \mathrm{~d} r^{2}+r^{2} \mathrm{~d} \Omega^{2}$.

Assuming the continuity of the metric functions $g_{t t}, g_{r r}$ and $\frac{\partial g_{t t}}{\partial r}$ at the boundary, we get

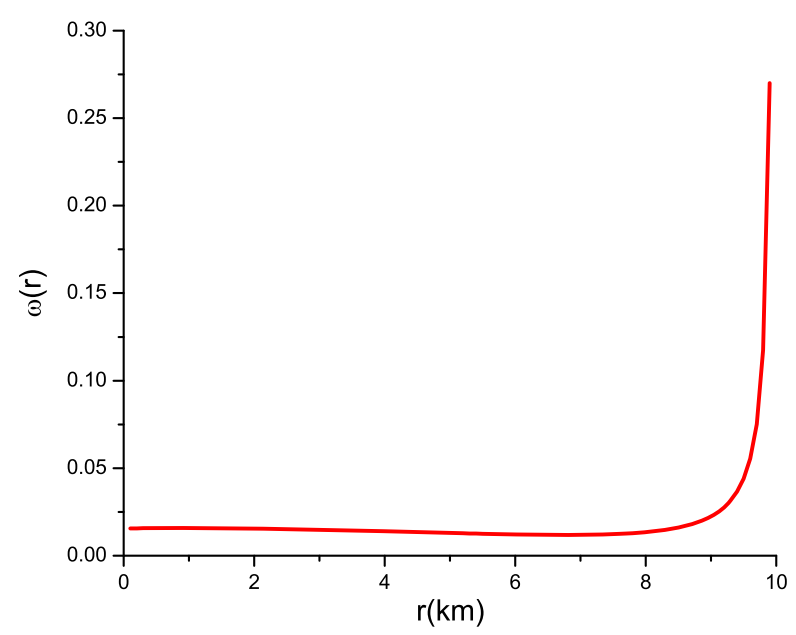

Fig. 4 Variation of dimensionless quantity $\omega(r)=\frac{p_{r}+2 p_{t}}{3 \rho}$, which determines a measure of the equation of state at the stellar interior of the strange star. We have taken the numerical values of the parameters as $b=10, R=26.25, A=25.999, C=0.05391$

$$
\left(1-\frac{b^{2}}{R^{2}}+4 \frac{b^{4}}{A^{4}}\right)=1-\frac{2 M}{b}
$$

and

$$
B^{2} \sin ^{2} \ln \sqrt{\frac{\sqrt{1-\frac{b^{2}}{R^{2}}+4 \frac{b^{4}}{A^{4}}}+2 \frac{b^{2}}{A^{2}}-\frac{1}{4} \frac{A^{2}}{R^{2}}}{C}}=\left(1-\frac{2 M}{b}\right) .
$$

Now from Eq. (11), we get the compactification factor

$\frac{M}{b}=\left(\frac{b^{2}}{2 R^{2}}-2 \frac{b^{4}}{A^{4}}\right)$.

\subsection{TOV equation}

For an anisotropic fluid distribution, the generalized TOV equation has the form

$\frac{\mathrm{d} p_{r}}{\mathrm{~d} r}+\frac{1}{2} v^{\prime}\left(\rho+p_{r}\right)+\frac{2}{r}\left(p_{r}-p_{t}\right)=0$.

Following [19], we write the above equation as

$$
-\frac{M_{G}\left(\rho+p_{r}\right)}{r^{2}} e^{\frac{\lambda-v}{2}}-\frac{\mathrm{d} p_{r}}{\mathrm{~d} r}+\frac{2}{r}\left(p_{t}-p_{r}\right)=0,
$$

where $M_{G}(r)$ is the gravitational mass inside a sphere of radius $r$ and is given by

$M_{G}(r)=\frac{1}{2} r^{2} e^{\frac{v-\lambda}{2}} v^{\prime}$

and $e^{\lambda(r)}=\left(1-\frac{r^{2}}{R^{2}}+4 \frac{r^{4}}{A^{4}}\right)^{-1}$, which can easily be derived from the Tolman-Whittaker formula and the Einstein field 


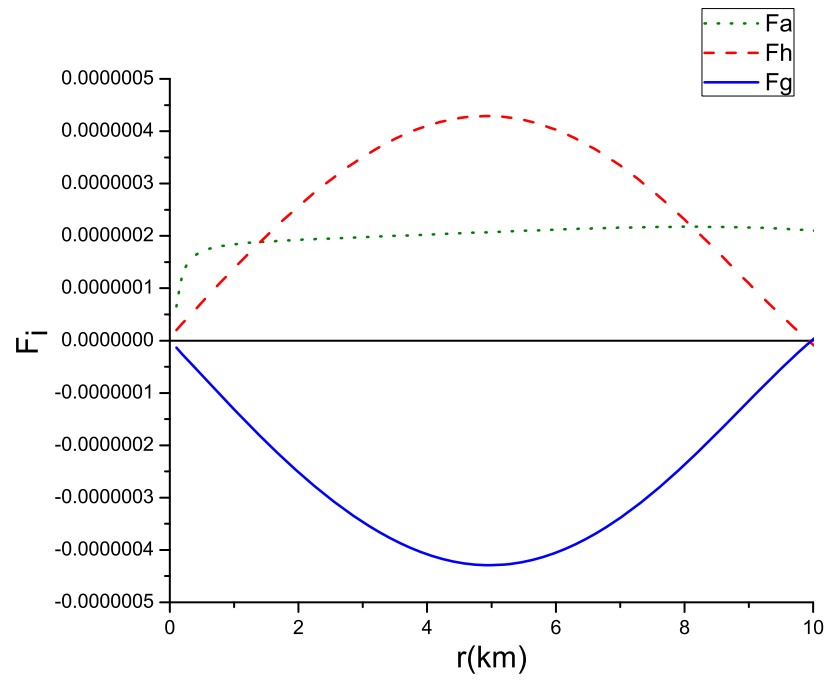

Fig. 5 Behaviours of pressure anisotropy and gravitational and hydrostatic forces at the stellar interior of strange star. We have taken the numerical values of the parameters as $b=10, R=26.25, A=$ $25.999, C=0.05391$

equations. The modified TOV equation describes the equilibrium condition for the strange star subject to an effective gravitational $\left(F_{g}\right)$ and effective hydrostatic $\left(F_{h}\right)$ plus another force due to the effective anisotropic $\left(F_{a}\right)$ nature of the stellar object as

$F_{g}+F_{h}+F_{a}=0$

where the force components are given by

$F_{g}=-\frac{1}{2} v^{\prime}\left(\rho+p_{r}\right)$

$F_{h}=-\frac{\mathrm{d} p_{r}}{\mathrm{~d} r}$

and

$F_{a}=\frac{2}{r}\left(p_{t}-p_{r}\right)$.

We plot (Fig. 5) the behaviours of the pressure anisotropy, and the gravitational and hydrostatic forces in the interior region, which shows clearly that the static equilibrium configurations do exist due to the combined effect of pressure anisotropy and gravitational and hydrostatic forces.

\subsection{Energy conditions}

All the energy conditions, namely the null energy condition (NEC), the weak energy condition (WEC), the strong energy condition (SEC) and the dominant energy condition (DEC), are satisfied at the centre $(r=0)$.

(i) NEC: $p_{0}+\rho_{0} \geq 0$,

(ii) $\mathrm{WEC}: p_{0}+\rho_{0} \geq 0, \quad \rho_{0} \geq 0$,

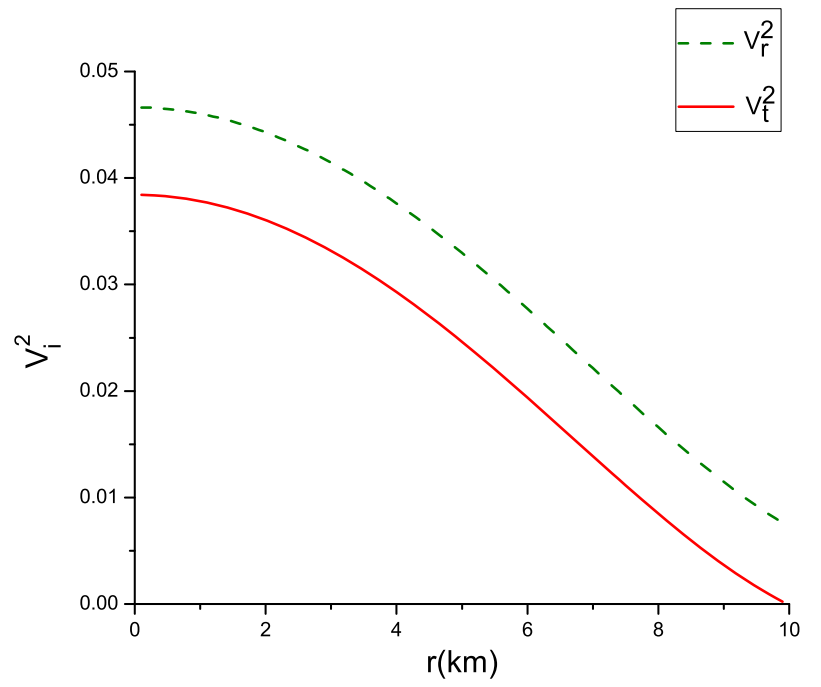

Fig. 6 Variation of the radial and transverse sound speed of the strange star. We have taken the numerical values of the parameters as $b=$ $10, R=26.25, A=25.999, C=0.05391$

(iii) SEC: $p_{0}+\rho_{0} \geq 0, \quad 3 p_{0}+\rho_{0} \geq 0$,

(iv) DEC: $\rho_{0}>\left|p_{0}\right|$.

We assume the numerical values of the parameters $R=$ 26.25, $A=25.999, C=0.05391$ to calculate the above energy conditions.

\subsection{Stability}

For a physically acceptable model, one expects that the velocity of sound should be within the range $0 \leq v_{s}^{2}=\left(\frac{\mathrm{d} p}{\mathrm{~d} \rho}\right) \leq 1$ $[14,20]$. According to Herrera's [14] cracking (or overturning) condition: The region for which radial speed of sound is greater than the transverse speed of sound is a potentially stable region.

In our case (anisotropic strange stars), we plot the radial and transverse sound speeds in Fig. 6 and observe that these parameters satisfy the inequalities $0 \leq v_{s r}^{2} \leq 1$ and $0 \leq$ $v_{s t}^{2} \leq 1$ everywhere within the stellar object. We also note that $v_{s t}^{2}-v_{s r}^{2} \leq 1$. Since, $0 \leq v_{s r}^{2} \leq 1$ and $0 \leq v_{s t}^{2} \leq 1$, we have $\left|v_{s t}^{2}-v_{s r}^{2}\right| \leq 1$. In Fig. 7, we have plotted $\mid v_{s t}^{2}-$ $v_{s r}^{2} \mid$. We notice that $v_{s t}^{2}<v_{s r}^{2}$ throughout the interior region. In other words, $v_{s t}^{2}<v_{s r}^{2}$ keeps the same sign everywhere within the matter distribution, i.e. no cracking will occur. These results show that our anisotropic compact star model is stable.

3.6 Mass-Radius relation and surface redshift

In this section, we study the maximum allowable massradius ratio in our model. According to Buchdahl [21], for a static spherically symmetric perfect fluid the allowable mass- 


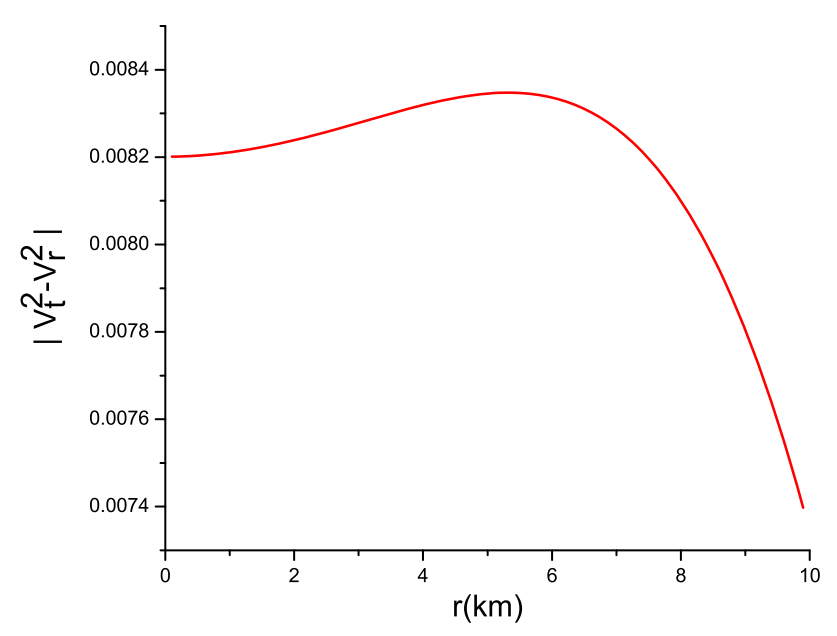

Fig. 7 Variation of $\left|v_{s t}^{2}-v_{s r}^{2}\right|$ of the strange star

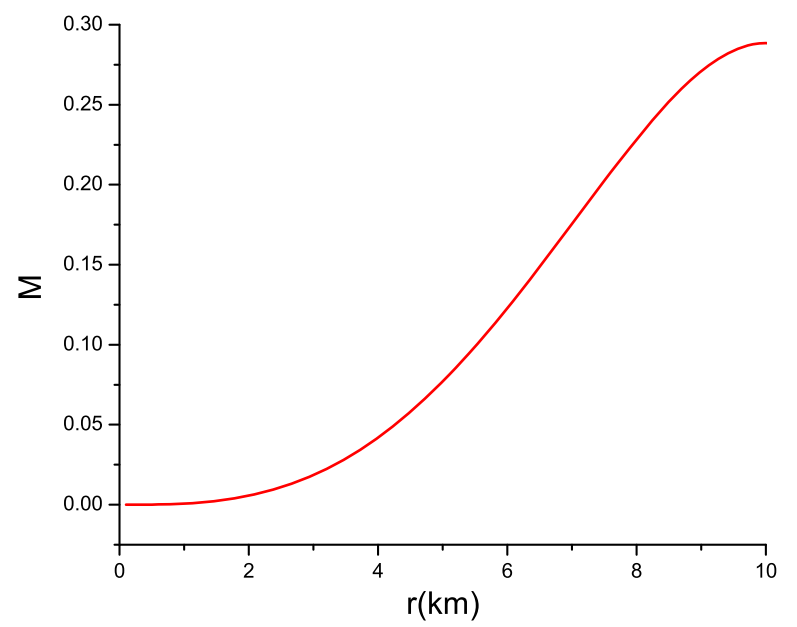

Fig. 8 Variation of the mass function of the strange star. We have taken the numerical values of the parameters as $b=10, R=26.25, A=$ 25.999, $C=0.05391$

radius ratio is given by $\frac{2 \text { Mass }}{\text { Radius }}<\frac{8}{9}$. Mak et al. [22] also gave more generalized expression. In our model the gravitational mass in terms of the energy density $\rho$ can be expressed as

$M=4 \pi \int_{0}^{b} \rho r^{2} \mathrm{~d} r=\frac{b}{2}\left[\frac{b^{2}}{R^{2}}-4 \frac{b^{4}}{A^{4}}\right]$.

The compactness of the star is given by

$u=\frac{M(b)}{b}=\frac{1}{2}\left[\frac{b^{2}}{R^{2}}-4 \frac{b^{4}}{A^{4}}\right]$.

The nature of the mass and compactness of the star from the centre are shown in Figs. 8 and 9.

The surface redshift $\left(Z_{s}\right)$ corresponding to the above compactness $(u)$ is obtained as

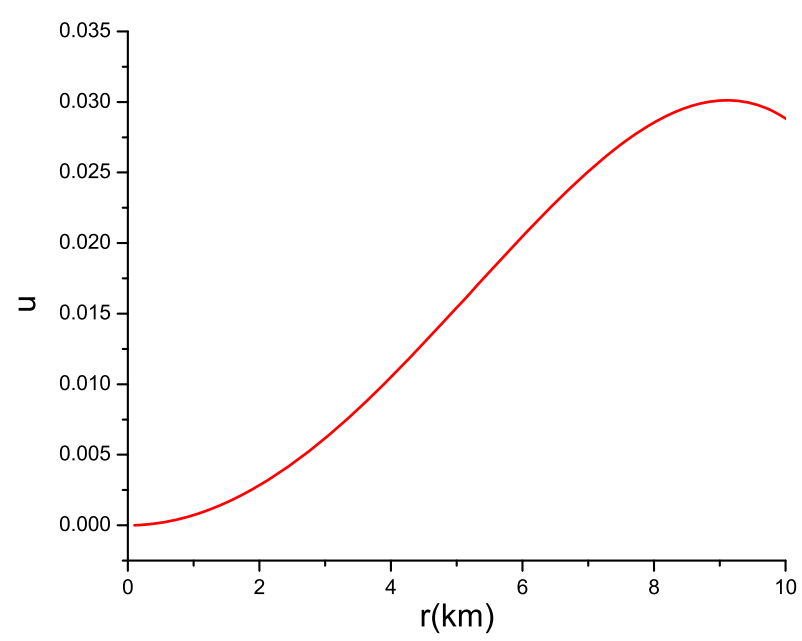

Fig. 9 Variation of the compactness of the strange star. We have taken the numerical values of the parameters as $b=10, R=26.25, A=$ 25.999, $C=0.05391$

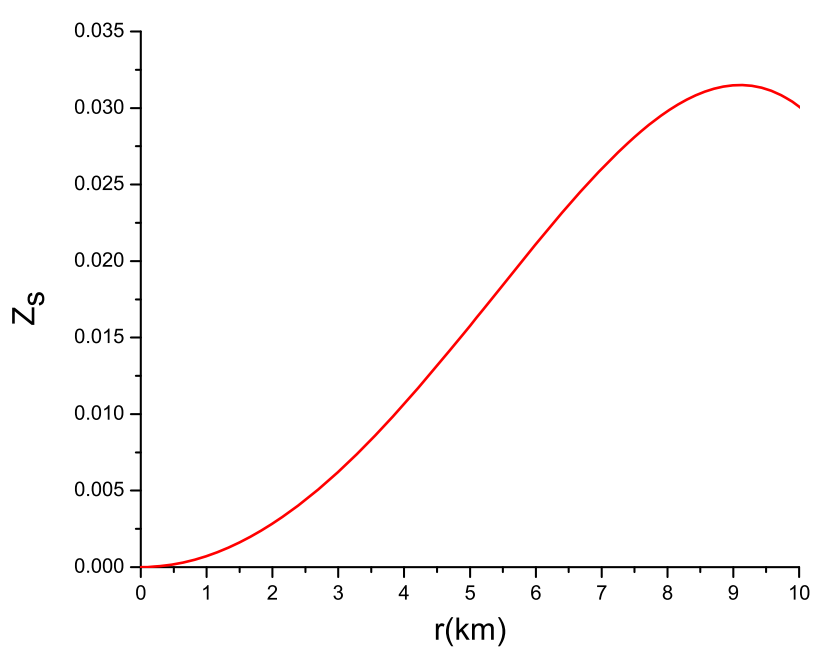

Fig. 10 Variation of the redshift function of the strange star. We have taken the numerical values of the parameters as $b=10, R=$ 26.25, $A=25.999, C=0.05391$

$1+Z_{s}=[1-(2 u)]^{-\frac{1}{2}}$

where

$Z_{s}=\frac{1}{\sqrt{1-\frac{b^{2}}{R^{2}}+4 \frac{b^{4}}{A^{4}}}}-1$.

Thus, the maximum surface redshift for the anisotropic strange stars of different radius could be found very easily from Fig. 10. We calculate the maximum surface redshift for our configuration using the numerical values of the parameters as $b=9.5, R=16.9, A=24.18$ and we get $Z_{s}=0.375$. The nature of the surface redshift of the star is shown in Fig. 10. 


\section{Conclusion}

In this work we have investigated the nature of anisotropic strange stars in the case of the low-mass X-ray binary $4 \mathrm{U}$ 1820-30 by making the following considerations: (a) The stars are anisotropic in nature i.e. $p_{r} \neq p_{t}$. (b) The spacetime of the strange stars can be described by the Tolman VII metric.

The results are quite interesting: (i) Though the radial pressure $\left(p_{r}\right)$ vanishes at the boundary $(r=b)$, the tangential pressure $\left(p_{t}\right)$ does not. However, at the centre of the star, its anisotropic behaviour vanishes. (ii) Our model is well stable according to the Herrera stability condition [14]. (iii) From the mass-radius relation, any interior features of the star can be evaluated.

Therefore, our overall features of anisotropic strange stars under the Tolman VII metric satisfy all physical requirements of a stable star.

It is to be noted that while solving Einstein's equations as well as for plotting, we have set $c=G=1$. Now, plugging $G$ and $c$ into the relevant equations, the values of the central density and surface density of our strange star turn out to be $\rho_{0}=0.55 \times 10^{15} \mathrm{gm} \mathrm{cm}^{-3}$ and $\rho_{b}=0.27 \times 10^{15}$ $\mathrm{gm} \mathrm{cm}{ }^{-3}$ for the numerical values of the parameters $b=$ $9.5, R=16.9, A=24.18$. Also, the mass of our strange star is calculated as $1.01 M_{\odot}$. Interestingly, we observe that the measurement of the mass, radius and central density of our strange star are almost consistent with the strange star in the low-mass X-ray binary 4U 1820-30 [18].

Recently, Cackett et al. [23] reported that the gravitational redshift of the strange star in the low-mass X-ray binary $4 \mathrm{U}$ 1820-30, based on the modelling of the relativistically broadened iron line in the X-ray spectrum of the source observed with Suzaku is $Z_{s}=0.43$. The surface redshift of our strange star with radius $9.5 \mathrm{~km}$ turns out to be 0.375 . This indicates that the measurement of the redshift of our strange star is nearly in compliance with the strange star in the low-mass X-ray binary $4 \mathrm{U}$ 1820-30.
Finally, we conclude by pointing out that spacetime comprising the Tolman VII metric with anisotropy may be used to construct a suitable model of a strange star in the low-mass X-ray binary $4 \mathrm{U} 1820-30$.

Acknowledgments MK, FR and SMH gratefully acknowledge support from IUCAA, Pune, India under Visiting Associateship under which a part of this work was carried out. FR is also thankful to UGC, for providing financial support under the research award scheme. We are grateful to the referee for valuable suggestions.

Open Access This article is distributed under the terms of the Creative Commons Attribution License which permits any use, distribution, and reproduction in any medium, provided the original author(s) and the source are credited.

Funded by $\mathrm{SCOAP}^{3}$ / License Version CC BY 4.0.

\section{References}

1. F. Rahaman et al., Gen. Relativ. Gravit. 44, 107 (2012)

2. F. Rahaman et al., Eur. Phys. J. C 72, 2071 (2012)

3. M. Kalam et al., Eur. Phys. J. C 72, 2248 (2012)

4. Sk. M. Hossein et al., Int. J. Mod. Phys. D 21, 1250088 (2012)

5. M. Kalam et al., Int. J. Theor. Phys. 52, 3319 (2013)

6. M. Kalam et al., Eur. Phys. J. C 73, 2409 (2013)

7. F. Lobo, Class. Quantum. Grav. 23, 1525 (2006)

8. K. Bronnikov, J.C. Fabris, Phys. Rev. Lett. 96, 251101 (2006)

9. E. Egeland, Compact Star (Trondheim, Norway, 2007)

10. I. Dymnikova, Class. Quantum. Gravit. 19, 725 (2002)

11. R.L. Bowers, E.P.T. Liang, Astrophys. J. 188, 657 (1974)

12. R. Ruderman, Rev. Astr. Astrophys. 10, 427 (1972)

13. A.I. Sokolov, JETP 52, 575 (1980)

14. L. Herrera, Phys. Lett. A 165, 206 (1992)

15. Stella et al. ApJ L 49, 315 (1987)

16. T.E. Strohmayer, E.F. Brown, ApJ 1045, 566 (2002)

17. R.C. Tolman, Phys. Rev. 55, 364 (1939)

18. T. Güver et al., ApJ 1807, 719 (2010)

19. J.P. de León, Gen. Relativ. Grav. 25, 1123 (1993)

20. H. Abreu, H. Hernandez, L.A. Nunez, Class. Quantum. Grav. 24, 4631 (2007)

21. H.A. Buchdahl, Phys. Rev. 116, 1027 (1959)

22. M.K. Mak, P.N. Dobson, T. Harko, Europhys. Lett. 55, 310 (2001)

23. E.M. Cackett et al., ApJ 674, 415 (2008) 